\title{
L'accesso vascolare per emodialisi nel terzo Millennio: fra realtà e utopia
}

\author{
Mauro Aloisi
}

\author{
U. O. di Nefrologia e Dialisi, Azienda USL 12 "Versilia", Regione Toscana, Pietrasanta, Lucca
}

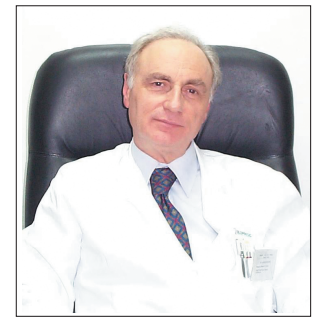

$\mathbf{F}$ in dagli albori dell'emodialisi extracorporea è apparso evidente che la sopravvivenza della tecnica era legata alla possibilità di disporre di un accesso alla circolazione sanguigna che fosse efficiente e duraturo, senza il quale la metodica sarebbe stata destinata esclusivamente al trattamento di pazienti uremici acuti.

Le due pietre miliari nella storia dell'accesso vascolare per emodialisi sono state lo shunt A-V esterno di silastic-teflon di Quinton-Scribner del 1960 e la fistola artero-venosa interna realizzata da Cimino-Brescia nel 1965. L'avvento delle protesi vascolari sintetiche, biologiche, semibiologiche ha ulteriormente ampliato la possibilità di realizzazione di un accesso vascolare. Infine l'utilizzo di cateteri venosi centrali tunnellizzati e muniti di manicotti in dacron, fino ai più recenti cateteri venosi centrali collegati a port totalmente impiantabili ha consentito di realizzare un accesso vascolare efficace e duraturo anche quando il patrimonio vascolare degli arti è di fatto esaurito o comunque non adeguato per altri tipi di accesso. In sintesi oggi disponiamo di varie soluzioni per realizzare un accesso vascolare adeguato ma, ciononostante, questo continua a rimanere il "tallone di Achille" dell'emodialisi extracorporea. Questa è la realtà.

L'utopia è a mio avviso ritenere di poter disporre di un "accesso vascolare ideale" che, in quanto tale, dovrebbe essere duraturo, privo di complicanze, indolore, in grado di assicurare un flusso ematico ottimale e costante, adatto per tutti i pazienti e, infine, anche di costo contenuto.

È certamente lecito, anzi doveroso, aspirare a realizzare un simile tipo di accesso vascolare e questa aspirazione è la "molla" per stimolare la ricerca. Ma nell'attesa che si realizzi l'accesso vascolare ideale del XXI secolo molto può essere fatto per utilizzare al meglio l'armamentario di cui attualmente disponiamo.

In questa ottica due sono a mio avviso le regole auree: 1) il risparmio del patrimonio vascolare; 2) l'attenta valutazione del paziente per ricercare l'accesso vascolare più idoneo o più adatto per quel paziente in relazione alle sue caratteristiche cliniche, alla situazione anatomica del patrimonio vascolare, alla sua età anagrafica e biologica, alle sue condizioni sociali e lavorative.

\section{Risparmio del patrimonio vascolare}

Il risparmio del patrimonio vascolare inizia ben prima che il paziente giunga alla fase terminale dell'uremia e coinvolge tutti gli operatori sanitari, medici e infermieri, con cui il paziente viene a contatto nel corso della sua storia clinica. Prelievi ematici ripetuti, iniezioni endovenose e infusioni di soluzioni flebosclerosanti possono danneggiare irreparabilmente il territorio venoso e renderlo inutilizzabile per l'allestimento di un accesso vascolare.

Al momento dell'inizio della dialisi per i "late referrals" è indispensabile il ricorso a cateteri venosi centrali temporanei; la scelta della sede di impianto del catetere è un momento particolarmente delicato per le conseguenze, anche gravi, che possono derivare al patrimonio vascolare del pa- 
ziente. Basti ricordare che, come riferito da Chimochowsky, all'incannulazione della succlavia possono far seguito elevate percentuali di stenosi $(58 \%)$. In questi casi qualsiasi utilizzo dell'arto superiore omolaterale per la creazione di un accesso vascolare con vasi nativi o protesico è precluso. Appare ovvio che nell'ottica del risparmio dei vasi saranno da preferirsi come sedi per il catetere temporaneo la vena femorale o la vena giugulare interna, avendo cura di mantenere il catetere per il minor tempo possibile e di utilizzare cateteri di materiale biocompatibile, sufficientemente morbidi.

\section{Attenta valutazione del paziente}

Ma risparmio del patrimonio vascolare significa anche utilizzare al meglio i vasi del paziente evitando interventi inutili, destinati all'insuccesso per le caratteristiche anatomiche dei vasi o per l'insufficiente esperienza dell'operatore chirurgo in rapporto alle difficoltà prevedibili. A questo scopo è certamente utile una valutazione ecografica e colordoppler preventiva che, in un approccio ideale, l'operatore chirurgo dovrebbe eseguire in prima persona o quantomeno presenziare per avere una visione diretta, un quadro preciso della situazione vascolare e quindi scegliere la strategia operatoria più adatta. In casi selezionati può essere utile far seguire all'esame ecocolordoppler una flebografia, in particolare quando si voglia valutare la pervietà dei vasi venosi centrali.

\section{L'idea del "team ideale"...}

Nasce quindi l'idea che siccome al momento non è disponibile un accesso vascolare ideale, potremmo almeno realizzare un "team ideale" per l'accesso vascolare composto dal nefrologo, dall'ecografista e dal chirurgo vascolare. Certamente "ideale" sarebbe che queste tre competenze si collocassero nella stessa unità operativa: cioè lo studio pre-operatorio e l'intervento chirurgico dovrebbero essere eseguiti da specialisti della stessa unità operativa, in pratica da nefrolo- gi con attitudini e competenze diverse. La collaborazione con il chirurgo vascolare dovrebbe essere riservata a situazioni cliniche che richiedono esperienza e competenza di livello superiore.

Nel caso in cui la chirurgia dell'accesso vascolare sia delegata al chirurgo vascolare, il nefrologo, che è il reale utilizzatore dell'accesso vascolare, dovrebbe comunque interagire con il chirurgo nella scelta del tipo di accesso vascolare da allestire, avendo egli una esatta conoscenza del quadro clinico del paziente e conoscendo le finalità dell'utilizzo dell'accesso stesso. Pertanto se a tutt'oggi non possiamo disporre dell'accesso vascolare "ideale", nel senso astratto e speculativo del termine, possiamo però già fín d'ora preparare il "team ideale" per lo studio e la preparazione dell'accesso vascolare. In questo modo potremo fornire al paziente, se non l'accesso ideale, almeno quello più adeguato alle sue condizioni cliniche, sociali, lavorative.

Il "team vascolare", valutato il paziente in tutti i suoi aspetti, tenendo sempre ben presente il principio basilare di risparmiare e quindi di utilizzare al meglio il patrimonio vascolare, sceglierà quello che per quel paziente, in quel momento rappresenta se non l'accesso vascolare "ideale" certamente il più idoneo.

\section{... e l'idea dell'Area Vasta}

È però evidente che il "team ideale" potrà svolgere al meglio il proprio compito soltanto se avrà un'adeguata preparazione ed esperienza "sul campo" che non sarà facile acquisire se limitata ai pazienti di un solo Centro dialisi, specie se piccolo. A questo scopo potrebbe essere utile creare Centri di chirurgia dell'accesso vascolare di riferimento, per "Area vasta", che comprendano vari Centri dialisi con un bacino di utenza complessivo di circa 1-1.5 milioni di abitanti. Questi Centri, di composizione multidisciplinare, dovrebbero, a mio avviso, essere coordinati da un nefrologo che dovrebbe avere anche il compito di intrattenere rapporti con $\mathrm{i}$ colleghi dei Centri dialisi afferenti per raccogliere tutte le informazioni cliniche, relative ai pazienti inviati utili nella scelta del tipo di accesso vascolare da realizzare.

Se tutto questo si potesse realizzare, se riuscissimo ad azzerare i "late referrals", se infine un'adeguata formazione del personale medico e infermieristico, che utilizza l'accesso vascolare, consentisse, nel rispetto delle linee guida, delle procedure e delle istruzioni operative, di mantenere adeguatamente ciò che è stato allestito nella sala operatoria, allora non avremmo costruito l'accesso vascolare ideale, ma almeno avremmo reso meno vulnerabile quel tallone di Achille e certamente avremmo ridotto i momenti di ansia, di angoscia e anche di sofferenza fisica dei pazienti dializzati.

m.aloisi@usl12.toscana.it 